\title{
Methods of passive and active mode locking using intracavity optical frequency mixing
}

\author{
Krassimir Stankov \\ Max-Planck-Institut für biophysikalische Chemie, Abteilung Laserphysik, Am Fassberg, D-3400 Göttingen, Federal Republic of Germany
}

Received August 17, 1988; accepted October 27, $1988^{\circ}$

\begin{abstract}
The mode-locking technique based on intracavity second-harmonic generation is extended to sum- and differencefrequency generation. Simultaneous passive mode locking of two different types of laser in a combined cavity is thus possible. By using the same principle, a laser may be actively mode locked by injection of a pulse train from another mode-locked laser.
\end{abstract}

Recently a promising new technique of passive mode locking based on intracavity second-harmonic generation in combination with an appropriate dichroic output coupler was proposed ${ }^{1}$ and demonstrated. ${ }^{2,3}$ This Letter shows that this technique can be extended to sum- and difference-frequency generation, which greatly expands its capabilities. In a manner analogous to that of Refs. 1-3, a nonlinear mirror featuring intensity-dependent reflection may be designed using a combination of a nonlinear medium for sum- or difference-frequency generation and a suitable trichroic mirror.

Consider the device, composed of the nonlinear medium, NLM, and the trichroic mirror, TM, shown in Fig. 1. Two beams at frequencies $\omega_{1}$ and $\omega_{2}$ are directed into the NLM. Mirror TM has reflectivities $R 1$ and $R 2$ at the two input frequencies $\omega_{1}$ and $\omega_{2}$ and reflectivity $R 3$ at the sum- or difference-frequency $\omega_{3}$. The principle of operation of the nonlinear optical device, composed of the NLM and the TM, is similar to that using second-harmonic generation. Consider the case of sum-frequency generation: in the first pass through the NLM the two input waves $\omega_{1}$ and $\omega_{2}$ generate the sum-frequency $\omega_{3}$. The radiation at $\omega_{3}$ is totally reflected by the TM, while the input waves $\omega_{1}$ and $\omega_{2}$ are only partially reflected. By adjusting the phase difference $\varphi=\varphi_{3}-\theta_{1}-\varphi_{2}\left(\varphi_{i}\right.$ are the phases of the interacting waves) to be $-\pi / 2$ after reflection by the TM, the interaction between the light waves is reversed ${ }^{4}$ and the reflected waves $\omega_{1}$ and $\omega_{2}$ are amplified at the expense of $\omega_{3}$. It is desirable to have the reflectivity of TM close to $100 \%$ at $\omega_{3}$ and to have lower reflectivities at the fundamental wavelengths. These conditions ensure that the amplification of either of the fundamental waves in the second pass through the NLM will be high. In this way a nonlinear reflection is obtained, manifested by an increase of the reflectivity when either of the incident wave intensities increases. By using this device, an intensity-dependent feedback may be provided, which is the basis of the mode locking.

The analysis of the nonlinear reflectivity is similar to that of Ref. 1, but it is now complicated since there are three interacting waves. The following parameters are introduced: photon conversion efficiency $\eta$ for the frequency $\omega_{1}$ and normalized photon numbers $n_{1}$ and $n_{2}$ for the two input waves and their ratio $\beta=$ $n_{1} / n_{2}$. We also use the abbreviations

$A=(1-\eta) R 1+\eta R 3, \quad B=(1 / \beta-\eta) R 2+\eta R 3$.

For sum-frequency generation, the following expressions for the nonlinear reflectivities $R 1_{\mathrm{NL}}$ and $R 2_{\mathrm{NL}}$ at the two input waves are derived:

$$
\begin{aligned}
R 1_{\mathrm{NL}}= & A\left(1-\operatorname{sn}^{2}\left\{\left[-\sqrt{\beta B} \cdot \mathrm{sn}^{-1}(\sqrt{\eta}, \sqrt{\beta})\right.\right.\right. \\
& \left.\left.\left.+\operatorname{sn}^{-1}(\sqrt{\eta R 3 / A}, \sqrt{A / B})\right], \sqrt{A / B}\right\}\right), \\
R 2_{\mathrm{NL}}= & \beta\left(B-A+R 1_{\mathrm{NL}}\right),
\end{aligned}
$$

where $\operatorname{sn}(u, m)$ and $\mathrm{sn}^{-1}(u, m)$ denote the Jacobian elliptic function and its inverse. Similar expressions are derived for difference-frequency generation. Although the derivation of the nonlinear reflection coefficients has been confined to plane monochromatic waves, the validity of these expressions can be extended up to the limit of the group-velocity dispersion effects. For sufficiently thin nonlinear crystals, a temporal response in the picosecond and subpicosecond time scales may be realized.

The variation of the normalized reflectivities at the fundamental frequencies $\omega_{1}$ and $\omega_{2}$ as a function of the photon conversion efficiency is shown in Fig. 2. In

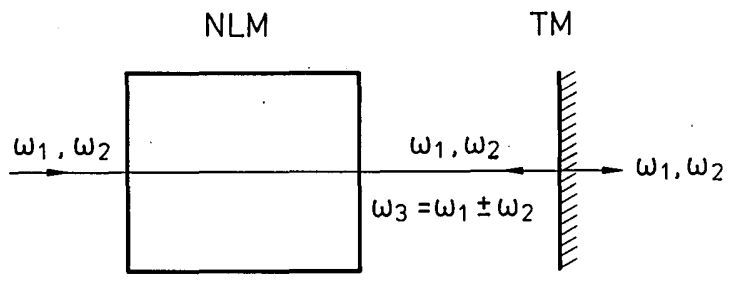

$R 1, R 2, R 3$

Fig. 1. Nonlinear mirror for two input waves with frequencies $\omega_{1}$ and $\omega_{2}$, based on sum- or difference-frequency generation in a NLM. The TM is a total reflector at $\omega_{3}$, with reflectivities $R 1$ and $R 2$ at $\omega_{1}$ and $\omega_{2}$. 


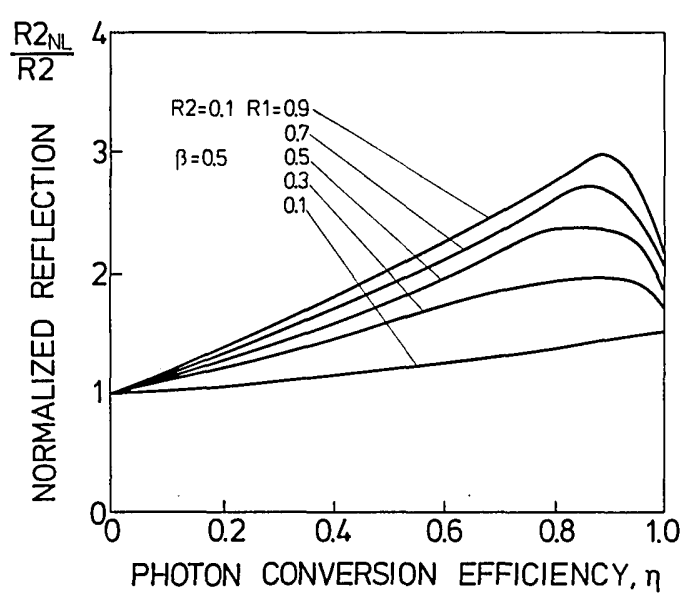

(a)

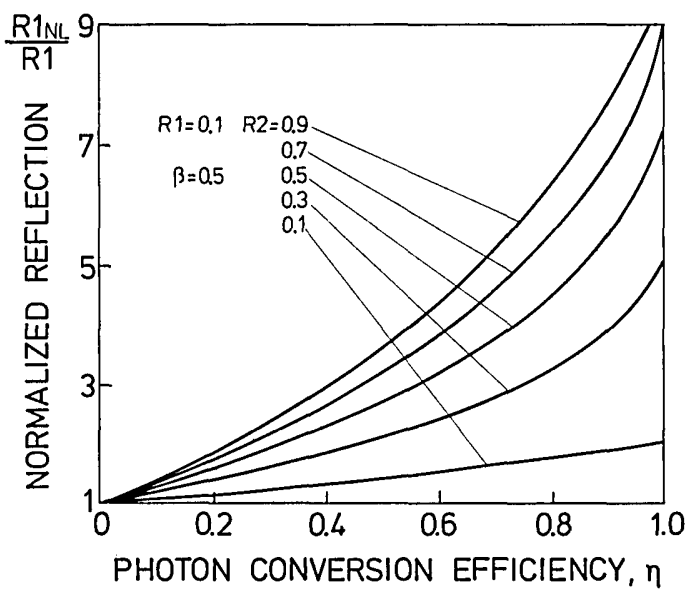

(b)

Fig. 2. (a) Normalized nonlinear reflection of the combination of the NLM and the TM at frequency $\omega_{1}$ as a function of the photon conversion efficiency for several values of the reflectivity $R 2$ at $\omega_{2}$. The TM is a total reflector at the sumfrequency radiation. (b) The same description as in (a) for frequency $\omega_{2}$.

this example the number of the incident photons at $\omega_{1}$ is half of that at $\omega_{2}(\beta=0.5)$. In Fig. 2(a) the reflectivity $R 1$ is fixed at 0.1 , and $R 2$ varies between 0.1 and 0.9 . The nonlinearity in reflection at $\omega_{1}$ increases when the reflectivity at the other frequency $\omega_{2}$ increases. This is reasonable, since the intensity of the differencefrequency $\omega_{1}=\omega_{3}-\omega_{2}$ amplified in the second pass as a reversed process of the sum-frequency generation in the first pass is proportional to the reflected intensities by the TM at $\omega_{2}$ and $\omega_{3}$. The same holds for the other frequency $\omega_{2}$, as shown in Fig. 2(b). A detailed analysis of this behavior will be published elsewhere.

Since the conversion efficiency $\eta$ is proportional to the intensities of the input waves, the reflectivities of this device $\omega_{1}$ and $\omega_{2}$ are also intensity dependent.

This device may be used for simultaneous mode locking of two different types of laser in a combined cavity, as shown in Fig. 3(a). The mode locker is the combination of the NLM and the TM shown within the dashed lines and is a common component of two lasers to be simultaneously mode locked. Since the reflection is intensity dependent at either of the laser wavelengths, amplitude discrimination and pulse shortening, similar to the case of the saturable absorber, will result in passive mode locking of both lasers. The cavity lengths of the two lasers should be matched. The principle of operation of the nonlinear mirror ensures inherently jitter-free generation of ultrashort pulses at the two laser wavelengths. Moreover, a pulse train at the sum or difference frequency is available on the left-hand side of the NLM, since total reconversion does not take place when $R 1$ and $R 2$ are less than unity.

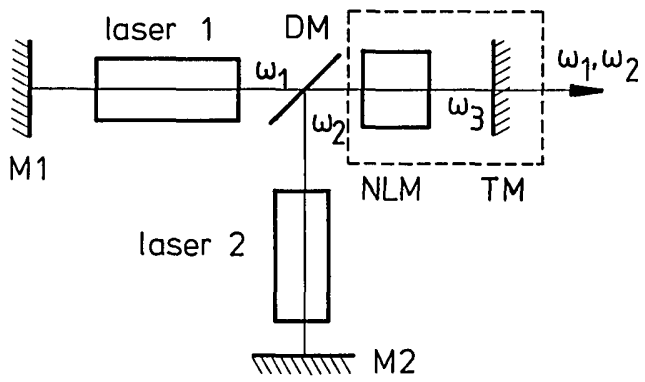

(a)

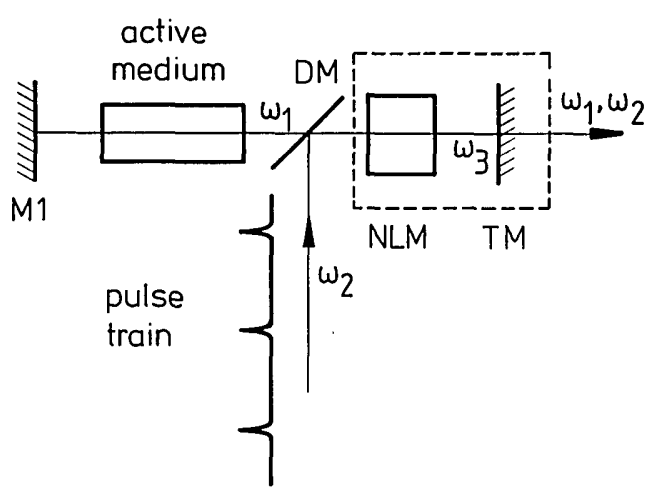

(b)

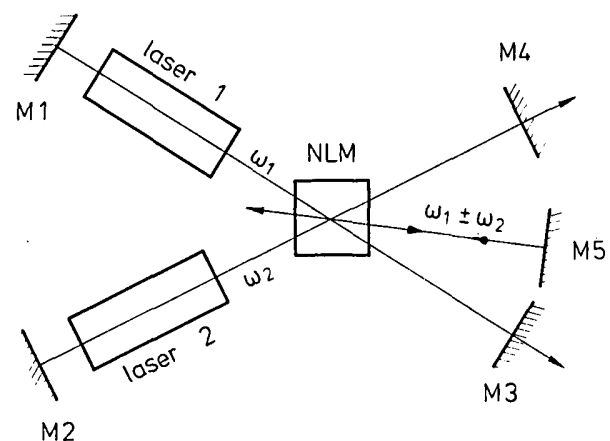

(c)

Fig. 3. (a) Arrangement for simultaneous mode locking of two lasers. M1 and TM, cavity mirrors of laser 1; M2 and TM, cavity mirrors of laser 2; DM, dichroic mirror or polarizer combining the two lasers beams. (b) Active mode locking of a laser by injecting a mode-locked laser pulse train. M1 and TM, laser cavity mirrors. (c) Arrangement for noncollinear mode locking: M1 and M3, M2 and M4, cavity mirrors of the two lasers; M5, total reflector at the mixed frequency. Active mode locking is analogous to that in (b). 
The easiest way to adjust the necessary phase difference $\varphi=-\pi / 2$ is to change the distance between the NLM and the TM, utilizing dispersion in air..$^{1-3}$

Active mode locking may be performed as shown in Fig. 3(b). An external pulse train from another modelocked laser is injected into the NLM by means of a suitable dichroic mirror DM. This will modulate the reflectivity of the nonlinear mirror, formed by NLM and TM, resulting in active mode locking. Here the term active is used in the sense that one of the cavity parameters (in this case, the reflectivity of the nonlinear mirror) is modulated externally at the proper frequency. The cavity length should match the injected pulse sequence.

Arrangements using noncollinear frequency mixing are also possible, as shown in Fig. 3(c). The two lasers have independent cavities, formed by mirrors M1 and M2 and M3 and M4. The laser axes cross at a point where a NLM for noncollinear frequency mixing is positioned. Mirror M5 reflects the mixed frequency back into the NLM. The advantage of this approach is that the two lasers may have independent mirrors, allowing easy optimization of the individual cavities. However, the optical path length between NLM and M5 should be maintained to within a fraction of a wavelength, i.e., an exceptional stability is required. Further application of this idea may be injection seeding in a manner similar to that shown in Fig. 3(b).

The proposed technique may be extended to other reversible nonlinear processes, e.g., Raman scattering in gases. However, an additional condition is imposed in this case, namely, $\omega_{1}-\omega_{2}=\Omega$ or $\omega_{2}-\omega_{1}=\Omega$, where $\Omega$ denotes the Raman shift. In some cases, e.g., deepUV lasers, this method may be preferable to the use of frequency mixing in crystalline nonlinear materials.

The advantages of the proposed mode-locking techniques are simplicity, durability, fast temporal response, and applicability to extremely wide spectral regions, ranging from deep UV to far IR. To demonstrate the capabilities of this new technique, an experiment is being prepared to mode lock simultaneously a long-pulse $\mathrm{XeCl}$ excimer laser and a $\mathrm{Nd}$ :YAG laser using difference-frequency generation.

The author thanks F. P. Schäfer, J. Jethwa, and B. Rácz for critical reading the manuscript. A research fellowship from the Alexander von Humboldt Foundation is gratefully acknowledged.

The author is on leave from the Department of Physics, Sofia University, 1126 Sofia, Bulgaria.

\section{References}

1. K. A. Stankov, Appl. Phys. B 45, 191 (1988).

2. K. A. Stankov, in Digest of Conference on Lasers and Electro-Optics (Optical Society of America, Washington, D.C., 1988), paper ThJ4.

3. K. A. Stankov and J. Jethwa, Opt. Commun. 66, 41 (1988).

4. J. A. Armstrong, N. Bloembergen, J. Ducuing, and P. S. Pershan, Phys. Rev. 127, 1918 (1962). 